\title{
EFFICIENCY ANALYSIS OF AODV, DSDV, DSR ROUTING PROTOCOLS
}

\author{
Prof.Ranjitha G \\ Department of CSE \\ Dayananda Sagar University, Karnataka, India
}

\section{Shakthi Priya J}

Department of CSE

Dayananda Sagar University, Karnataka, India

\begin{abstract}
MANET stands for Mobile Ad-Hoc Network, they consist of mobile nodes that offer a routable networking environment in the link layer. The nodes are connected wirelessly in a self-healing, self-configured network that have no fixed infrastructure or centralized management. Routing in MANET's is a very inconvenient process as wireless links in this network are highly prone to error and go down frequently because of the mobility of nodes. Therefore, it is a highly researched topic around the world, and to overcome such difficulty many routing protocols have been suggested around the world to improve its efficiency. Some protocols used in this paper are Ad-hoc On-demand Distance Vector (AODV), Destination Sequenced Distance Vector (DSDV), and Dynamic Source Routing. This paper hopes to summarize all the recent work done on MANET Routing protocols and the network simulator tool.
\end{abstract}

Keywords - MANET, Proactive and Reactive routing protocols, Multicasting, DSDV, AODV, DSR

\section{INTRODUCTION}

Mobile ad hoc networks are autonomously self-organized networks without infrastructure support [5]. Nodes are allowed to move freely in an arbitrary fashion therefore there are chances that unpredictable rapid topological changes may occur in the network. MANET network can move data in any direction and communicates with any node present in the network. Every mobile device in MANET is connected for a given session. Where every single each device behaves as a router to transfer pockets of data to other mobile devices, by analyzing best routing algorithms when we get a lot of nodes included in the network our main task is to analyze for the efficient routing protocol to proceed with the network building. This paper presents effective analysis of the routing protocol by considering various characteristics. Our study includes comparison of three main and most widely used routing protocol AODV DSDV and DSR. In efficiency

\author{
Sathwik J R \\ Department of CSE \\ Dayananda Sagar University, Karnataka, India \\ Sushmitha G \\ Department of CSE \\ Dayananda Sagar University, Karnataka, India
}

analysis we are considering various factors and parameters like number of nodes, Bandwidth etc. Different classifications of MANETs are,

- Vehicular Ad-Hoc Network (VANET)

- In this technology moving cars are connected as nodes for transporting messages to other moving cars in nodes.

- This network is very useful in providing various information, like details about the traffic at certain paths to the connected nodes.

- Nodes are connected within the range of $100 \mathrm{~m}$ to $300 \mathrm{~m}$ geographical area for communication.

- Internet Based Mobile Ad-Hoc Network (iMANET)

- Mobile nodes in this technique are also selforganized

- This is mostly used in commercial applications.

- Intelligent Vehicular Ad-Hoc Network (InVANET)

- Vehicle2Vehicle and vehicle2RSU communications are established. It offers high security and provides easy efficient communication

A routing protocol dictates how routers must communicate among themselves. The main reason for them to communicate is to distribute information that enables them to select routes between various nodes present on a network. Routers perform the traffic directing functions, data packets are transferred across networks among routers until they reach their destination node. Routing algorithms are used to determine the specific choice of route to transfer data. Each router will be having knowledge only of networks attached to it. A routing protocol shares this information first among immediate neighbours, and then throughout. Routers gain knowledge of the topology of the network through the network. Routing protocols have the ability to dynamically adjust to instant changing conditions such as connection disabling and components failure and route data around obstructions is what gives the Internet its fault tolerance and high availability. 


\section{International Journal of Engineering Applied Sciences and Technology, 2021 \\ Vol. 5, Issue 11, ISSN No. 2455-2143, Pages 181-184 \\ Published Online March 2021 in IJEAST (http://www.ijeast.com)}

Few specific characteristics of routing protocols are:

- Ability in avoiding routing loops.

- Ability to select preferred routes, using information about hop costs, the time they require to reach routing convergence, scalability, relay multiplexing and cloud access framework parameters.

- Multilayer interfacing is also employed.

- Technical standards documents containing other routing protocols are defined.

Classification of Routing Protocols in MANET's

To properly understand and study the features, similarities, and differences between different routing protocols, it is really important to understand how these protocols are grouped together. Researchers use classification methods to understand different routing protocols by easy comparison. Routing protocols in MANETs are broadly classified into three sub groups,

\section{Proactive Routing Protocols}

Proactive Routing Protocols, also known as 'table driven' routing protocol maintains information on all nodes throughout the network, even if they are not required. Therefore each node registers a route to all the other nodes in the network, rendering such information available at all times ensures quick transition of nodes throughout the network. The core feature of a proactive routing protocol is its routing manager that is responsible for handling (searching, adding, updating and removing) existing and new routes attending to the various situations of the network. When a change in network topology occurs its change should be notified throughout the network, this feature forces this protocol to keep a track of all the changes made to the network irrespective of whether data traffic exists or not. Therefore, the overhead to maintain up to date information regarding the network is very high. A consequence of this is that the cost of maintaining the network increases considerably if there is a frequent change in network topology, whereas if the network activity is very low the actual information about topology change is rendered useless.

\section{Reactive Routing Protocols}

Reactive Routing Protocols, or more popularly known as 'on demand' routing protocol. The routers find out the best separate path for a particular node during the time of sending that data packet to that node. The main advantage of Reactive Routing Protocol is that it takes off the overhead since we don't want to occasionally swamp the network with routing tables. But, compared with Proactive, nodes in reactive take more time because source nodes start searching for a path to destination when the data packet arrives in the subnet. Few Reactive algorithms are DSR and AODV.
Hybrid Routing Protocol

Often the features offered in the above two routing protocols separately is not sufficient, to overcome this challenge a Hybrid Routing Protocol is used, which is a combination of both Proactive and Reactive protocols that achieve better efficiency. Hybrid Routing Protocols offer the best of both worlds by utilizing reactive routing in large scale networks (In order to decrease control overhead) and proactive routing (In order to decrease delay) in small scale networks. Hybrid protocols usually serve activated nodes via reactive flooding.

\section{NS - 2 Simulation Tool}

A simulation is a visual representation of a physical phenomenon using mathematical modelling. Network Simulator 2 (NS 2), is a discrete event simulator targeted at networking research. Its current development is supported through DARPA with SAMAN and through NSF with CONSER, both in collaboration with other researchers including ACIRI.

\section{ROUTING PROTOCOLS}

Ad-hoc On Demand Distance Vector Routing (AODV) Protocol

AODV, a reactive routing protocol, is an improvement of the DSDV routing protocol. For route discovery, a route request packet (RREQ) is broadcasted whenever a node has a packet to transmit to the destination [6]. Intermediate nodes record the address of the adjacent nodes from which the first copy of the broadcast copy is received. A reverse path can also be established as all the information is stored in a route table. If duplicate RREQ packets are received, it discards them keeping only one intact. In AODV a continuous process of link failure and route discovery phase is attempted by the source node and the adjacent nodes until the desired destination is reached.

\section{Destination Sequenced Distance Vector (DSDV) Protocol}

In DSDV protocol every node maintains routing in sequence to all known destinations [7]. Every node contains path information about all the possible destinations, irrespective of it being required or not to the node. Node destination details are present in the Routing table which gets exchanged periodically with the help of such routing tables in each mobile node, the packets can be transmitted between nodes of a MANET. Each node of the ad hoc network needs to update the routing table with latest details either periodically or when 


\section{International Journal of Engineering Applied Sciences and Technology, 2021 \\ Vol. 5, Issue 11, ISSN No. 2455-2143, Pages 181-184 \\ Published Online March 2021 in IJEAST (http://www.ijeast.com)}

important new information is available. This is done so to maintain the consistency of the dynamically changing topology of the ad hoc network. Every entry in routing table will specify the destination address, number of nodes distance of destination node etc.

\section{Dynamic Source Routing (DSR) Protocol}

DSR is a wholly reactive routing protocol, it makes inquiries for the route from source to destination, when there is a need. It allows a node to dynamically discover a source route across multiple network hops to any destination [2]. If node wants to send data to another node, it searches its cache for a route. If a route is found, it puts the required information to the packet and sends it to the destination. During the course of travelling on the packet, if the link breaks, an error message is sent to the source code. If a route is not found, then the source node discovers the route by sending by sending ROUTE REQUEST to the destination nodes.

\section{CONCLUSION}

This paper is a summary of comprehensive research on MANET's and its various routing protocols which are broadly classified as Proactive, Reactive and Hybrid protocols. Routing protocols are chosen depending on the flow of network traffic between nodes. The overall answer as to the efficiency of each protocol is still highly debatable and researched throughout the world and it depends on various factors such as mobility of nodes and traffic patterns of networks. Therefore, it is very difficult to assume or predict the best routing protocol as one solution cannot be applied to all the situations and even if we could it might not be optimal.

\section{REFERENCES}

[1] Kaur, Sandeep \& Beri, Rydhm \& Singh, Satbir. (2016). A Survey on Mobile Ad Hoc Network (MANET). International Journal for Scientific Research and Development (IJSRD), Vol 4, no. 7, pp. 1064-1066, Online ISSN: 2321-0613

[2]Ravi Kumar1, N. Geethanjali2. (2013) a Literature Survey of Routing Protocols in MANETs, International Journal of Science and Research (IJSR), India Online ISSN: 2319-7064

[3] A. Nadeem and M. P. Howarth, "A Survey of MANET Intrusion Detection \& Prevention Approaches for Network Layer Attacks," in IEEE Communications Surveys \& Tutorials, vol. 15, no. 4, pp. 2027-2045, Fourth Quarter 2013, doi: 10.1109/SURV.2013.030713.00201.

[4] Chamkaur Singh, Vikas Gupta, Gurmeet Kaur. "A Review Paper on Introduction to MANET", International Journal of Engineering Trends and Technology (IJETT), V11 (1), 38-43 May 2014. ISSN: 2231-5381.
[5] G.Vijaya, Kumar \& Y.Vasudeva, Reddy \& Dr.M.Nagendra. (2010). Current Research Work on Routing Protocols for MANET: A Literature Survey. International Journal on Computer Science and Engineering. 2.

[6] Ashtiani, Hossein \& Pour, Hamed \& Nikpour, Mohsen. (2010). A Survey of MANET Routing Protocols in LargeScale and Ordinary Networks.

[7] Puri, Swati \& Arora, Vishal. (2014). Routing Protocols in MANET: A Survey. International Journal of Computer Applications. 96. 7-12. 10.5120/16852-6718.

[8] Yasmin Jahir, Mohammed Atiquzzaman, Hazem Refai, Anirudh Paranjothi, Peter G. LoPresti,

Routing protocols and architecture for disaster area network: A survey,Ad Hoc Networks,Volume 82, 2019 ,Pages 114,ISSN 1570-8705

[9] M. Naseem and C. Kumar, "EDSDV: Efficient DSDV routing protocol for MANET," 2013 IEEE International Conference on Computational Intelligence and Computing Research, Enathi, India, 2013, pp. 1-4, doi: 10.1109/ICCIC.2013.6724265.

[10] H. Moudni, M. Er-rouidi, H. Mouncif and B. El Hadadi, "Performance analysis of AODV routing protocol in MANET under the influence of routing attacks," 2016 International Conference on Electrical and Information Technologies (ICEIT), Tangiers, Morocco, 2016, pp. 536-542, doi: 10.1109/EITech.2016.7519658.

[11] Almazok, S.A., Bilgehan, B. A novel dynamic source routing (DSR) protocol based on minimum execution time scheduling and moth flame optimization (MET-MFO). $J$ Wireless Com Network 2020, 219 (2020).

[12] CYNTHIA, PRUDHVI KRISHNA SAGUTURU, C et al. A survey on MANET protocols in wireless sensor networks. International Journal of Engineering \& Technology, [S.1.], v. 7, n. 2.31, p. 1-3, may 2018. ISSN 2227-524X.

[13] D. N. Patel, S. B. Patel, H. R. Kothadiya, P. D. Jethwa and R. H. Jhaveri, "A survey of reactive routing protocols in MANET," International Conference on Information Communication and Embedded Systems (ICICES2014), Chennai, India, 2014, pp. 1-6, doi: 10.1109/ICICES.2014.7033833.

[14] G. Mohandas, S. Silas and S. Sam, "Survey on routing protocols on mobile adhoc networks," 2013 International Mutli-Conference on Automation, Computing, Communication, Control and Compressed Sensing (iMac4s), Kottayam, India, 2013, pp. 514-517, doi: 10.1109/iMac4s.2013.6526467.

[15] Avdhut Suryakant Bhise, Prof. R.N. Phursule, 2013, The Survey of Routing Protocols in Wireless Network, INTERNATIONAL JOURNAL OF ENGINEERING RESEARCH \& TECHNOLOGY (IJERT) Volume 02, Issue 09 (September 2013) 
International Journal of Engineering Applied Sciences and Technology, 2021 Vol. 5, Issue 11, ISSN No. 2455-2143, Pages 181-184

Published Online March 2021 in IJEAST (http://www.ijeast.com) 